\title{
Correction to: Choosing the target difference and undertaking and reporting the sample size calculation for a randomised controlled trial - the development of the DELTA2 guidance
}

William Sones ${ }^{1}$, Steven A. Julious², Joanne C. Rothwell², Craig Robert Ramsay ${ }^{3}$, Lisa V. Hampson ${ }^{4,5}$, Richard Emsley ${ }^{6}$, Stephen J. Walters ${ }^{2}$, Catherine Hewitt ${ }^{7}$, Martin Bland ${ }^{7}$, Dean A. Fergussonn, Jesse A. Berlin ${ }^{9}$, Doug Altman ${ }^{1}$, Luke David Vale ${ }^{10}$ and Jonathan Alistair Cook ${ }^{1 *}$

Correction to: Trials (2018) 19:542

https://doi.org/10.1186/s13063-018-2887-x

Following publication of the original article [1], we have been notified of a few mistakes:

- Figure 1:

- "1351 titles and abstracts" should read "1322 titles and abstracts"

- "Stat Biopharm Res" value should be "8" instead of "18"

- "Stat Med" value should be "226" instead of "74"

- "Stat Methods Med Res" value should be "74" instead of "226"

- Results section, Stage 5, first paragraph, the phrase:

- "It was endorsed by the MRC-NIHR Methodology Advisory Group on March 12, 2018 , with minor updating of references and the final version produced on April 18, 2017" should read

- "It was endorsed by the MRC-NIHR Methodology Advisory Group on March 12, 2018 , with minor updating of references and the final version produced on April 18, 2018".

\section{Author details}

${ }^{1}$ Centre for Statistics in Medicine, Nuffield Department of Orthopaedics, Rheumatology and Musculoskeletal Sciences, University of Oxford, Botnar Research Centre, Nuffield Orthopaedic Centre, Windmill Rd, Oxford OX3 7LD, UK. ${ }^{2}$ Medical Statistics Group, ScHARR, The University of Sheffield, Regent Court, 30 Regent Street, Sheffield S1 4DA, UK. ${ }^{3}$ Health Services Research Unit, University of Aberdeen, Health Sciences Building, Foresterhill, Aberdeen AB25 2ZD, UK. ${ }^{4}$ Department of Mathematics and Statistics, Lancaster University, Lancaster LA1 4YF, UK. ${ }^{5}$ Statistical Methodology and Consulting, Novartis Pharma AG, Basel, Switzerland. 'Department of Biostatistics and Health Informatics, Institute of Psychiatry, Psychology and Neuroscience, King 's College London, De Crespigny Park, Denmark Hill, London SE5 8AF, UK. ${ }^{7}$ Department of Health Sciences, Seebohm Rowntree Building, University of York, Heslington, York YO10 5DD, UK. ${ }^{8}$ Clinical Epidemiology Program, Ottawa Hospital Research Institute, 501 Smyth Road, Box 201B, Ottawa, ON $\mathrm{K} 1 \mathrm{H}$ 8L6, Canada. ${ }^{9}$ Johnson \& Johnson, One J\&J Plaza, New Brunswick, NJ 08933, USA. ${ }^{10}$ Health Economics Group, Institute of Health and Society, Newcastle University, Newcastle upon Tyne, UK.

Published online: 24 October 2019

\section{Reference}

1. Sones $W$, et al. Choosing the target difference and undertaking and reporting the sample size calculation for a randomised controlled trial - the development of the DELTA2 guidance. Trials. 2018;19:542. https://doi.org/10. 1186/s13063-018-2887-x.

* Correspondence: jonathan.cook@ndorms.ox.ac.uk

${ }^{1}$ Centre for Statistics in Medicine, Nuffield Department of Orthopaedics,

Rheumatology and Musculoskeletal Sciences, University of Oxford, Botnar Research Centre, Nuffield Orthopaedic Centre, Windmill Rd, Oxford OX3 7LD, UK

Full list of author information is available at the end of the article

(c) The Author(s). 2019 Open Access This article is distributed under the terms of the Creative Commons Attribution 4.0 International License (http://creativecommons.org/licenses/by/4.0/), which permits unrestricted use, distribution, and reproduction in any medium, provided you give appropriate credit to the original author(s) and the source, provide a link to the Creative Commons license, and indicate if changes were made. The Creative Commons Public Domain Dedication waiver (http://creativecommons.org/publicdomain/zero/1.0/) applies to the data made available in this article, unless otherwise stated. 